\section{Cough and Pleuritic Chest Pain in a Middle-aged Man}

\section{Khajotia RR*}

Department of Internal Medicine and Pulmonology, International Medical University, Seremban, Malaysia, and Consultant Pulmonologist, Hospital Tuanku Ja'afar, Seremban, Malaysia

\section{Clinical Scenario}

A 55-year-old male patient, chronic smoker, comes to the hospital with complaints of cough, yellowish expectoration and haemoptysis, accompanied by a sharp, stabbing pain in the right axillary region which is aggravated on taking a deep breath and on coughing. On examination, the trachea is shifted to the right side, a dull note on percussion is heard in the right 2 nd-5th intercostal spaces anteriorly, extending to the axilla and right interscapular region. Coarse crepitations, bronchial breath sounds and increased vocal resonance are heard in the same region anteriorly and in the right axilla. A chest radiograph is taken.
Q1) Interpret the chest radiographic findings.

Q2) Based on the history and radiological findings, what is the most likely clinical diagnosis?

Q3) State three possible underlying aetiological causes for the above condition.

Q4) Besides chest radiography, which other investigations would you perform in this patient? (Figure 1)

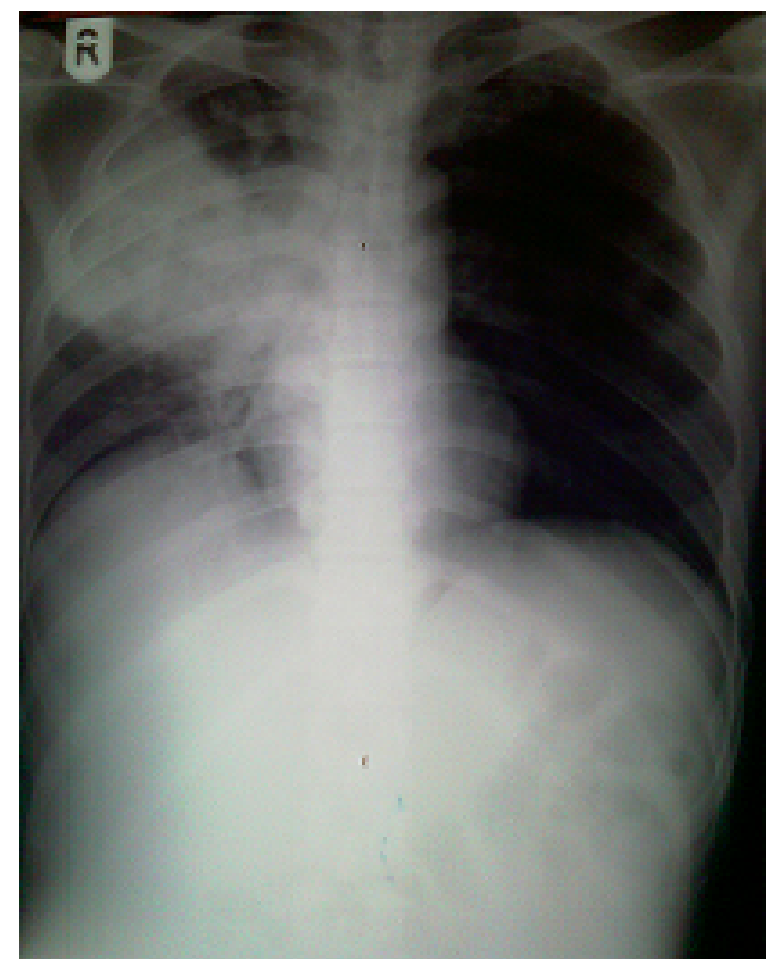

Figure 1: A chest radiograph

\footnotetext{
*Corresponding author: Rumi R Khajotia, MBBS, Associate Professor in Internal Medicine and Pulmonology, International Medical University, Seremban, Malaysia, and Consultant Pulmonologist, Hospital Tuanku Ja'afar, Seremban, Malaysia E-mail: rumi@imu.edu.my

Received December 28, 2015; Accepted December 29, 2015; Published December 30, 2015

Citation: Khajotia RR (2015) Cough and Pleuritic Chest Pain in a Middle-aged Man. J Clin Respir Dis Care 1: i001. doi: 10.4172/ JCRDC.1000i001

Copyright: ( 2015 Khajotia RR. This is an open-access article distributed under the terms of the Creative Commons Attribution License, which permits unrestricted use, distribution, and reproduction in any medium, provided the original author and source are credited.
} 
A1) Chest radiograph shows the trachea shifted to the right side. There is a homogenous well-defined opacity in the right mid-zone which extends up to the chest wall laterally and merges with the hilum medially. The right hemi diaphragm is elevated. The left lung fields are clear.

A2) Collapse-consolidation of the middle lobe and part of the upper lobe of the right lung.

A3) Bronchogenic Carcinoma

Pulmonary Tuberculosis

Bacterial infection

A4) Full blood count, Sputum for AFB smear and culture, bacterial culture and antibiotic sensitivity and sputum cytology for malignant cells

Erythrocyte sedimentation rate (ESR)

Mantoux test

Tumour markers

High-resolution (HRCT) chest scan

Percutaneous CT-guided lung biopsy (if required) Fibreoptic bronchoscopy

\section{Discussion}

This case highlights the difficulties in making a clinical diagnosis in a middle-aged patient with cough and pleuritic chest pain. While a consolidation is apparent on the right side on clinical and radiological examination, the discerning physician will also realize that the trachea is shifted to the same side as the lesion (right side) and the right hemi diaphragm too is significantly elevated.

This would make bronchogenic carcinoma a very likely possibility. In this condition, due to a proximal bronchial obstruction by a tumor, the distal lung parenchyma gets infected and inflamed and a consolidation along with an accompanying collapse develops. In case of lung cancer this collapse is usually severe enough to cause the trachea to get pulled to the same side as the lesion. The elevated hemi diaphragm can be explained by the collapsed lung pulling it upwards and/or a phrenic nerve palsy occurring due to the phrenic nerve getting entrapped by tumor infiltration.

Pulmonary tuberculosis is also a differential diagnosis in this patient. While tuberculosis pneumonia can explain the presence of the consolidation, the trachea may be pulled to the same side as the lesion due to fibrosis and scarring, which is commonly associated with pulmonary tuberculosis. However, an elevated hemi diaphragm is uncommon in this condition. Entrapment of the phrenic nerve by chronic fibrosis and scarring which occurs during tuberculosis, is however a possibility in this condition.

Sputum examination, tumor markers, HRCT chest scan, fine needle aspiration biopsy of the lung, bronchoscopy, thoracoscopy and thoracentesis are usually the diagnostic investigations done to arrive at a definitive diagnosis in such a patient. In this patient, phrenic nerve palsy was confirmed by fluoroscopy of the chest and bronchoscopy revealed an infiltrating mass in the right middle-lobe bronchus. Bronchoscopic biopsy showed a non-small cell lung carcinoma. 